TITLE:

\title{
Third-order nonlinear effects on femtosecond X-ray-absorption near-edge spectra
}

\author{
$\operatorname{AUTHOR}(\mathrm{S})$ :
}

Kitamura, Hikaru

\section{CITATION:}

Kitamura, Hikaru. Third-order nonlinear effects on femtosecond X-rayabsorption near-edge spectra. Physical Review A 2020, 102 (2): 023120.

ISSUE DATE:

2020-08

URL:

http://hdl.handle.net/2433/261143

\section{RIGHT:}

(c) 2020 American Physical Society; 許諾条件に基づいて掲載しています $\circ$ 
PHYSICAL REVIEW A 102, 023120 (2020)

\title{
Third-order nonlinear effects on femtosecond $x$-ray-absorption near-edge spectra
}

\author{
Hikaru Kitamura $\odot$ \\ Department of Physics, Kyoto University, Sakyo-ku, Kyoto 606-8502, Japan
}

(Received 10 March 2020; accepted 6 August 2020; published 31 August 2020)

\begin{abstract}
Reduced density matrix equations combined with a cluster molecular-orbital method are formulated to describe resonant core-valence electron excitation by an intense femtosecond x-ray pulse. The electron-hole interaction is treated by the time-dependent unrestricted Hartree-Fock approximation supplemented by a screening correction in the Born approximation. Nonlinear $K$-shell absorption spectra of metallic copper are thereby computed. Numerical results indicate that, in the third-order nonlinear regime, where the fraction of excited electrons increases in proportion to the x-ray intensity, the absorption spectra above the $K$ edge undergo shifts toward high-energy side. The origin of this third-order nonlinearity can be traced to a negative renormalization of the core orbital energy due to strong electron-hole attraction, which offers a sharp contrast to the usual optical nonlinearity due simply to the increment of upper-state populations. For higher intensities, broadening of the absorption edge and saturation of absorption are predicted. These trends are qualitatively consistent with nonlinear spectroscopy experiments using the SACLA x-ray free-electron laser.
\end{abstract}

DOI: 10.1103/PhysRevA.102.023120

\section{INTRODUCTION}

When a laser pulse with sufficiently high intensity is incident on a material, its linear optical constants such as the refractive index and absorption coefficient are modified, which in turn affects the propagation of the pulse itself [1]. In such a nonlinear self-action process, the complex electric susceptibility $\chi_{\mathrm{e}}(\omega)$, defined as the ratio between the frequency-dependent electric polarization density $\tilde{\mathbf{P}}(\omega)$ and the incident electric field $\tilde{\mathbf{E}}(\omega)$, depends on laser intensity [1]. From a perturbation-theoretic point of view, the lowest-order correction to the linear optical constant arises from the thirdorder nonlinearity [1-4]. As a dominant contribution to the third-order nonlinearity of a metal in the visible frequency range, modification of electron population near the Fermi level, which results from resonant interband excitation and/or generation of hot electrons, has been proposed $[5,6]$.

Recent experiments using $\mathrm{x}$-ray free-electron lasers (XFEL's) demonstrated nonlinear absorption of an x-ray pulse transmitting through metallic films [7-9]. In these experiments, intense resonant excitation of $K$-shell electrons to the conduction band results in saturable absorption $[8,9]$ or change in $\mathrm{x}$-ray-absorption near-edge spectra [7]. In corelevel excitation, localized core holes are created and their strong perturbation can induce anomalies in the x-ray spectra even in low-intensity (linear-response) regime [10]. It is an outstanding issue of nonlinear optics to elucidate the influence of core holes on the shape of absorption spectrum as the $\mathrm{x}$-ray intensity is gradually elevated.

In this paper, we formulate reduced density-matrix (RDM) equations, combined with a cluster molecular-orbital method, to simulate time evolutions of nonlinear electric polarization associated with resonant core-level excitation by a femtosecond x-ray pulse. As a numerical example, complex suscepti- bilities and the corresponding $K$-shell absorption spectra of metallic copper are computed for various $\mathrm{x}$-ray intensities by focusing especially on the third-order nonlinear regime. The laser pulse duration is assumed to be less than $10 \mathrm{fs}$ so that we ignore those energetic processes as hot plasma formation, collisional ionization, Auger heating, and so on. We thus find that renormalization of the core orbital energy serves as a dominant source of nonlinearity, giving rise to a blueshift of absorption spectra as the intensity increases. This mechanism is a consequence of large electron-hole attraction energy inherent in a localized core orbital, which is absent in usual optical nonlinearity caused by excitation of delocalized valence electrons.

\section{REDUCED DENSITY MATRIX (RDM) EQUATIONS}

Our theoretical framework is outlined as follows: We consider a finite cluster consisting of $N$ atoms that mimics a local portion of the bulk crystal $[10,11]$. The positions of the nuclei $\mathbf{R}_{\mu}(\mu=1, \ldots, N)$ are fixed. We first calculate one-electron wave functions and energies of the cluster in the ground state by the molecular-orbital (MO) method. We then introduce a one-electron RDM in spectral representation by regarding these MOs as an orthonormal basis set. Electric polarization induced by an x-ray pulse can be obtained by solving time evolution equations for the off-diagonal elements of the RDM. Fourier transform of the induced polarization gives the nonlinear complex susceptibility, whose imaginary part yields the absorption spectrum. The x-ray electric field will be treated classically throughout this work.

\section{A. Cluster molecular orbitals}

The electronic structures of the cluster are computed self-consistently by the semiempirical unrestricted 
Hartree-Fock (UHF) MO method based on the neglect of diatomic differential overlap (NDDO) approximation; the details are described elsewhere [12]. We adopt a spin-unrestricted formalism, in which the wave function $\psi_{k \sigma}(\mathbf{r})$ and energy $\varepsilon_{k \sigma}$ of each MO depend on the spin index $\sigma=\alpha, \beta$. The core orbitals $\psi_{c \mu, \sigma}(\mathbf{r}) \quad\left(c=1, \ldots, N_{\text {core }}\right)$ localized at atom $\mu(=1, \ldots, N)$ and their energy eigenvalues $\varepsilon_{c \mu, \sigma}$ are assumed to be identical to those of an isolated atom. Each valence $\mathrm{MO} \psi_{v \sigma}(\mathbf{r})\left(v=1, \ldots, N_{\mathrm{MO}}\right)$ is expressed as a linear combination of $N_{\mathrm{vo}}$ valence atomic orbitals (AO's) as $\psi_{v \sigma}(\mathbf{r})=\sum_{\mu=1}^{N} \sum_{k=1}^{N_{\mathrm{vo}}} C_{k \mu, v}^{\sigma} \phi_{k}\left(\mathbf{r}-\mathbf{R}_{\mu}\right)$, where the total number of MO's is $N_{\mathrm{MO}}=N N_{\mathrm{vo}}$. The AO's $\left\{\phi_{k}(\mathbf{r})\right\}$ consist of occupied and unoccupied (virtual) states, including discrete pseudostates in continuum. The NDDO approximation neglects an overlap of AO's centered at different sites as

$$
\phi_{k}\left(\mathbf{r}-\mathbf{R}_{\mu}\right) \phi_{k^{\prime}}\left(\mathbf{r}-\mathbf{R}_{\mu^{\prime}}\right)=0 \text { for } \mu \neq \mu^{\prime} .
$$

The resultant MO's $\left\{\psi_{c \mu, \sigma}(\mathbf{r})\right\}$ and $\left\{\psi_{v \sigma}(\mathbf{r})\right\}$ form a set of (approximately) orthonormal basis functions, which are employed to evaluate the matrix elements of dipole transitions and Coulomb repulsion integrals as we shall explain later in Sec. II C.

\section{B. Slowly varying envelope approximation (SVEA)}

The slowly varying envelope approximation (SVEA) is a standard procedure to analyze ultrafast laser pulse phenomena [13]. The electric field associated with the applied x-ray pulse at time $t$ and position $\mathbf{r}$ in the cluster is assumed to take the form

$$
\mathbf{E}(\mathbf{r}, t)=\frac{1}{2}\left[\mathbf{E}_{v}(t) e^{i\left(\mathbf{k}_{v} \cdot \mathbf{r}-\omega_{v} t\right)}+\mathbf{E}_{v}^{*}(t) e^{-i\left(\mathbf{k}_{v} \cdot \mathbf{r}-\omega_{v} t\right)}\right] .
$$

The complex field envelope $\mathbf{E}_{v}(t)$ varies slowly in time compared to the $\mathrm{x}$-ray frequency $\omega_{\nu}$; its spatial variation inside the cluster is neglected. As far as we exclude high harmon- ics, we may likewise express the local induced polarization density as

$$
\mathbf{P}(\mathbf{r}, t)=\frac{1}{2}\left[\mathbf{P}_{v}(t) e^{i\left(\mathbf{k}_{v} \cdot \mathbf{r}-\omega_{v} t\right)}+\mathbf{P}_{v}^{*}(t) e^{-i\left(\mathbf{k}_{v} \cdot \mathbf{r}-\omega_{v} t\right)}\right] .
$$

We consider a nearly resonant excitation of an electron from core level $c$ to valence level $v$ by the X-ray field (2). The complex off-diagonal element of the one-electron RDM relevant to this process is decomposed as

$$
\begin{aligned}
& \left\langle\rho_{v, c \mu}^{\sigma}(\mathbf{r}, t)\right\rangle \\
& =\frac{1}{2}\left[\left\langle\rho_{v, c \mu}^{\sigma}(t)\right\rangle_{\nu} e^{i\left(\mathbf{k}_{v} \cdot \mathbf{r}-\omega_{v} t\right)}+\left\langle\rho_{c \mu, v}^{\sigma}(t)\right\rangle_{\nu}^{*} e^{-i\left(\mathbf{k}_{v} \cdot \mathbf{r}-\omega_{v} t\right)}\right] \\
& \quad+\left\langle\rho_{v, c \mu}^{\sigma}(\mathbf{r}, t)\right\rangle_{0} .
\end{aligned}
$$

The quantity $\left\langle\rho_{v, c \mu}^{\sigma}(t)\right\rangle_{\nu}$ is directly connected with resonantly induced electric polarization, whereas the nonresonant component $\left\langle\rho_{c \mu, v}^{\sigma}(t)\right\rangle_{v}$ is ignored by the rotating-wave approximation [13]. The component $\left\langle\rho_{v, c \mu}^{\sigma}(t)\right\rangle_{0}$ related to orbital deformation [14] will also be neglected so far as the excitation is not so strong. The SVEA assumption enables one to eliminate the rapidly oscillating exponential factors common to Eqs. (2)-(4); slow dynamics of the remaining envelope functions will be studied in the following sections.

\section{Time evolutions of RDM}

A variety of representations are applicable to general RDM formalisms for optical phenomena [15]. In this work, we adopt spectral representation and describe the dynamics of RDM in the space spanned by the cluster MO's introduced in Sec. II A. Since these MO's are eigenstates of the unperturbed cluster Hamiltonian, the RDM is diagonal initially $(t=0)$. Off-diagonal elements of the RDM describe mixing of different one-electron states after an X-ray pulse is applied. By rearranging the time-dependent unrestricted Hartree-Fock (TDUHF) equations for one-particle RDM within SVEA $[12,14,16]$, we can derive the following closed set of equations for one-particle RDM:

$$
\begin{aligned}
\frac{\partial\left\langle\rho_{v, c \mu}^{\sigma}(t)\right\rangle_{\nu}}{\partial t}= & i\left\{\omega_{v}-\frac{\varepsilon_{v \sigma}-\varepsilon_{c \mu \sigma}}{\hbar}-\frac{1}{\hbar}\left[\left(\tilde{\mathcal{E}}_{v v}^{\sigma}(t)\right)_{0}-\left(\tilde{\mathcal{E}}_{c \mu, c \mu}^{\sigma}(t)\right)_{0}\right]+i \Gamma\right\}\left\langle\rho_{v, c \mu}^{\sigma}(t)\right\rangle_{\nu} \\
& -\frac{i}{\hbar}\left[\left(\tilde{\mathcal{E}}_{v, c \mu}^{\sigma}(t)\right)_{v}^{\mathrm{sc}}-\mathbf{d}_{v, c \mu}^{\sigma} \cdot \mathbf{E}_{v}(t)\right]\left[f_{c \mu \sigma}(t)-f_{v \sigma}(t)\right] \\
\frac{\partial f_{c \mu \sigma}(t)}{\partial t}= & \left.\frac{1}{2 \hbar} \operatorname{Im}\left\{\sum_{v=1}^{N_{\mathrm{MO}}}\left[\left(\tilde{\mathcal{E}}_{v, c \mu}^{\sigma}(t)\right)_{v}^{s c *}-\mathbf{d}_{v, c \mu}^{\sigma *} \cdot \mathbf{E}_{v}^{*}(t)\right]\left\langle\rho_{v, c \mu}^{\sigma}(t)\right\rangle_{v}\right\}+\frac{\partial f_{c \mu \sigma}(t)}{\partial t}\right]_{\text {Auger }}, \\
\frac{\partial f_{v \sigma}(t)}{\partial t}= & \frac{1}{2 \hbar} \operatorname{Im}\left\{\sum_{c=1}^{N_{\text {core }}} \sum_{\mu=1}^{N}\left[\left(\tilde{\mathcal{E}}_{v, c \mu}^{\sigma}(t)\right)_{v}^{\mathrm{sc}}-\mathbf{d}_{v, c \mu}^{\sigma} \cdot \mathbf{E}_{v}(t)\right]\left\langle\rho_{v, c \mu}^{\sigma}(t)\right\rangle_{v}^{*}\right\}
\end{aligned}
$$

The diagonal elements of the RDM $f_{c \mu \sigma}(t)$ and $f_{v \sigma}(t)$ stand for the populations of core and valence MOs, respectively, at time $t$, which are assumed to be slowly varying. Electron screening beyond the UHF approximation is incorporated into $\left(\tilde{\mathcal{E}}_{v, c \mu}^{\sigma}(t)\right)_{v}^{\text {sc }}$ within the Born approximation (BA) [12] to be described below. We ignore X-ray induced core-core and valence-valence transitions so that the corre- sponding off-diagonal RDM's are neglected. When we set $\partial f_{c \mu \sigma}(t) / \partial t=\partial f_{v \sigma}(t) / \partial t=0$, the resultant Eq. (5a) reduces to the linear-response equation studied earlier [12]. The corevalence dipole transition matrix is defined as

$$
\mathbf{d}_{v, c \mu}^{\sigma} \equiv-e \int d \mathbf{r} \psi_{v \sigma}^{*}(\mathbf{r}) \mathbf{r} \psi_{c \mu \sigma}(\mathbf{r})
$$


which is to be evaluated by expanding $\psi_{v \sigma}(\mathbf{r})$ in terms of AO's and adopting the NDDO approximation (1). The last term on the right-hand side of Eq. (5b) accounts for change in core-level population due to Auger decay $[17,18]$. It is assumed that the emitted Auger electrons escape freely from the system.

Many-body effects due to the formation of core holes may be classified into final-state interactions and cascaded processes. The final-state interactions, such as scattering of an excited electron by the core hole (excitonic effect [19]) and valence electron redistribution (screening), are taken into account through the screened self-energy matrix [12]:

$$
\left(\tilde{\mathcal{E}}_{v, c \mu}^{\sigma}(t)\right)_{\nu}^{\mathrm{sc}}=-\sum_{v^{\prime}=1}^{N_{\mathrm{MO}}} W_{v, v^{\prime}, c \mu, c \mu}^{\sigma \sigma}\left\langle\rho_{v^{\prime}, c \mu}^{\sigma}(t)\right\rangle_{\nu} .
$$

Here, the matrix element of screened Coulomb interaction,

$$
\begin{aligned}
& W_{v, v^{\prime}, c \mu, c \mu}^{\sigma \sigma} \\
& =V_{v, v^{\prime}, c \mu, c \mu}^{\sigma \sigma}+\sum_{v_{1}, v_{2}=1}^{N_{\mathrm{MO}}} \sum_{\sigma_{1}=\alpha, \beta} V_{v v^{\prime} v_{1} v_{2}}^{\sigma \sigma_{1}} \chi_{v_{1} v_{2}, v_{2} v_{1}}^{\sigma_{1}(0)} V_{v_{2}, v_{1}, c \mu, c \mu}^{\sigma_{1} \sigma}
\end{aligned}
$$

accounts for a process such that an electron excited to state $v$ is scattered immediately to $v^{\prime}$ by the core hole $c$ at site $\mu$. The first term on the right-hand side of (8) stands for the bare
Coulomb repulsion integral

$$
\begin{aligned}
V_{k k^{\prime} k_{1} k_{2}}^{\sigma \sigma_{1}} \equiv & \int d \mathbf{r} \int d \mathbf{r}^{\prime} \psi_{k \sigma}^{*}(\mathbf{r}) \psi_{k^{\prime} \sigma}(\mathbf{r}) \\
& \times \frac{e^{2}}{\left|\mathbf{r}-\mathbf{r}^{\prime}\right|} \psi_{k_{1} \sigma_{1}}^{*}\left(\mathbf{r}^{\prime}\right) \psi_{k_{2} \sigma_{1}}\left(\mathbf{r}^{\prime}\right),
\end{aligned}
$$

whereas

$$
\chi_{v_{1} v_{2}, v_{2} v_{1}}^{\sigma_{1}(0)}=\frac{f_{v_{1} \sigma_{1}}(0)-f_{v_{2} \sigma_{1}}(0)}{\varepsilon_{v_{1} \sigma_{1}}-\varepsilon_{v_{2} \sigma_{1}}}
$$

represents the static response function accounting for screening action of valence electrons [12]. Expressions (7) and (8) can be derived from the equation of motion for twoparticle RDM in BA as given in Ref. [12]. The summation on the right-hand side of Eq. (7) produces a mixing of different off-diagonal elements $\left(v^{\prime} \neq v\right)$ of the RDM; the $v^{\prime}=v$ term acts to reduce the bare excitation energy $\varepsilon_{v \sigma}-\varepsilon_{c \mu \sigma}$ in Eq. (5a) [12]. It was shown in the previous work [12] that the bare scattering matrix element $V_{v, v^{\prime}, c \mu, c \mu}^{\sigma \sigma}$ in Eq. (8) considerably overestimates the excitonic enhancement of absorption; the screening correction represented by the last term of Eq. (8) is essentially important. The matrix elements (9) should be evaluated within the NDDO approximation (1) to keep consistency with the formalism in Sec. II A.

Formation of a core hole yields a renormalization of energy levels due to Coulomb interaction, which is described by a diagonal element of the self-energy matrix in (5a) [14]. The core and valence self-energy matrices can be expressed, respectively, in the TDUHF approximation as

$$
\begin{aligned}
\left(\tilde{\mathcal{E}}_{c \mu, c \mu}^{\sigma}(t)\right)_{0} \approx & \sum_{c^{\prime}=1}^{N_{\text {core }}} \sum_{\mu^{\prime}=1}^{N} \sum_{\sigma^{\prime}=\alpha, \beta}\left(V_{c \mu, c \mu, c^{\prime} \mu^{\prime}, c^{\prime} \mu^{\prime}}^{\sigma \sigma^{\prime}}-\delta_{\sigma \sigma^{\prime}} \delta_{\mu \mu^{\prime}} V_{c \mu, c^{\prime} \mu, c^{\prime} \mu, c \mu}^{\sigma \sigma}\right)\left[f_{c^{\prime} \mu^{\prime} \sigma^{\prime}}(t)-f_{c^{\prime} \mu^{\prime} \sigma^{\prime}}(0)\right] \\
& +\sum_{v=1}^{N_{\mathrm{MO}}} \sum_{\sigma^{\prime}} V_{c \mu, c \mu, v, v}^{\sigma \sigma^{\prime}}\left[f_{v \sigma^{\prime}}(t)-f_{v \sigma^{\prime}}(0)\right], \\
\left(\tilde{\mathcal{E}}_{v v}^{\sigma}(t)\right)_{0} \approx & \sum_{c=1}^{N_{\text {core }}} \sum_{\mu=1}^{N} \sum_{\sigma^{\prime}=\alpha, \beta} V_{v, v, c \mu, c \mu}^{\sigma \sigma^{\prime}}\left[f_{c \mu \sigma^{\prime}}(t)-f_{c \mu \sigma^{\prime}}(0)\right] \\
& +\sum_{v^{\prime}=1}^{N_{\mathrm{MO}}} \sum_{\sigma^{\prime}=\alpha, \beta}\left(V_{v v v^{\prime} v^{\prime}}^{\sigma \sigma^{\prime}}-\delta_{\sigma \sigma^{\prime}} V_{v v^{\prime} v^{\prime} v}^{\sigma \sigma}\right)\left[f_{v^{\prime} \sigma^{\prime}}(t)-f_{v^{\prime} \sigma^{\prime}}(0)\right] .
\end{aligned}
$$

Note that $\left(\tilde{\mathcal{E}}_{c \mu, c \mu}^{\sigma}(0)\right)_{0}=\left(\tilde{\mathcal{E}}_{v v}^{\sigma}(0)\right)_{0}=0$ by definition. The first line on the right-hand side of Eq. (11a) describes a shift of one-electron energy for orbital $c \mu$ induced by formation of a hole in orbital $c^{\prime} \mu^{\prime}$; the electron-hole interaction between these two orbitals is described by the Hartree term $V_{c \mu, c \mu, c^{\prime} \mu^{\prime}, c^{\prime} \mu^{\prime}}^{\sigma \sigma^{\prime}}$ and the exchange term $V_{c \mu, c^{\prime} \mu, c^{\prime} \mu, c \mu}^{\sigma \sigma}$, where the latter is relevant only for parallel-spin pairs. The second line on the right-hand side of (11a) represents a shift induced by population change in the valence band, where the core-valence exchange term $V_{c \mu, v, v, c \mu}^{\sigma \sigma}$ has been neglected since it is small. The valence-level renormalization (11b) can be interpreted in a similar way. The energy- level renormalization may be viewed as a cascaded process $[2,4]$ that starts to develop after the population change is initiated.

Initial conditions to Eqs. (5a)-(5c) are

$$
\begin{aligned}
& \left\langle\rho_{v, c \mu}^{\sigma}(0)\right\rangle_{v}=0, \quad f_{c \mu \sigma}(0)=1, \\
& f_{v \sigma}(0)=\left\{\begin{array}{l}
1, v=1, \ldots, N_{\text {val }}^{\sigma} \\
0, v=N_{\mathrm{val}}^{\sigma}+1, \ldots, N_{\mathrm{MO}},
\end{array}\right.
\end{aligned}
$$

where $N_{\text {val }}^{\sigma}$ designates the total number of valence electrons with spin $\sigma$. 


\section{Electric polarization and absorption coefficient}

With the knowledge of the off-diagonal elements of RDM, the induced electric polarization per unit volume can be obtained by taking their average inside the cluster:

$$
\mathbf{P}_{v}(t)=\frac{n_{\text {atom }}}{N} \sum_{c=1}^{N_{\text {core }}} \sum_{\mu=1}^{N} \sum_{v=1}^{N_{\text {MO }}} \sum_{\sigma=\alpha, \beta} \mathbf{d}_{c \mu, v}^{\sigma}\left\langle\rho_{v, c \mu}^{\sigma}(t)\right\rangle_{\nu} .
$$

The damping factor $\Gamma$ in (5a) ensures that $\mathbf{P}_{v}(t) \rightarrow 0$ for $t$ sufficiently longer than the pulse duration. We can then take the Fourier transform into the frequency space,

$$
\tilde{\mathbf{P}}_{v}(\omega) \equiv \int_{0}^{\infty} d t \mathbf{P}_{v}(t) e^{i \omega t}, \quad \tilde{\mathbf{E}}_{v}(\omega) \equiv \int_{0}^{\infty} d t \mathbf{E}_{v}(t) e^{i \omega t}
$$

If we assume that the x-ray is polarized along the $x$ direction as $\mathbf{E}_{v}(t)=E_{v}(t) \hat{\mathbf{x}}$ and consider the polarization component along that direction $\mathbf{P}_{v}(t)=P_{v}(t) \hat{\mathbf{x}}$, the nonlinear complex electric susceptibility can be calculated as

$$
\begin{aligned}
& \chi_{\mathrm{e}}(\omega)=\frac{\tilde{P}_{\nu}\left(\omega-\omega_{\nu}\right)}{\tilde{E}_{\nu}\left(\omega-\omega_{v}\right)} . \\
& \langle H(t)\rangle=\sum_{v=1}^{N_{\mathrm{MO}}} \sum_{\sigma=\alpha, \beta}\left[\varepsilon_{v \sigma}-\frac{1}{2} W_{v v \sigma}+\frac{1}{2}\left(\tilde{\mathcal{E}}_{v v}^{\sigma}(t)\right)_{0}\right] f_{v \sigma}(t) \\
& +\sum_{c=1}^{N_{\text {core }}} \sum_{\mu=1}^{N} \sum_{\sigma=\alpha, \beta}\left[\varepsilon_{c \mu \sigma}-\frac{1}{2} W_{c \mu, c \mu, \sigma}+\frac{1}{2}\left(\tilde{\mathcal{E}}_{c \mu, c \mu}^{\sigma}(t)\right)_{0}\right] f_{c \mu \sigma}(t) \\
& +\frac{1}{2} \operatorname{Re}\left\{\sum_{v=1}^{N_{\mathrm{MO}}} \sum_{c=1}^{N_{\text {core }}} \sum_{\mu=1}^{N} \sum_{\sigma=\alpha, \beta}\left[-\mathbf{d}_{v, c \mu, \sigma} \cdot \mathbf{E}_{\nu}(t)+\frac{1}{2}\left(\tilde{\mathcal{E}}_{v, c \mu}^{\sigma}(t)\right)_{v}^{\mathrm{sc}}\right]\left\langle\left.\rho_{v, c \mu}^{\sigma}(t)\right|_{\nu} ^{*}\right\},\right.
\end{aligned}
$$

The absorption coefficient is thus obtained as

$$
\kappa_{\mathrm{abs}}(\omega)=\frac{4 \pi \omega}{c} \operatorname{Im} \chi_{\mathrm{e}}(\omega)
$$

Electric polarization is related to some important physical quantities derived from the RDM equations. The instantaneous core-valence excitation rate per unit volume, for instance, can be calculated with the aid of Eq. (5c) as

$$
w_{\text {exc }}(t) \equiv \frac{n_{\text {atom }}}{N} \sum_{v=1}^{N_{\mathrm{MO}}} \sum_{\sigma=\alpha, \beta} \frac{\partial f_{v \sigma}(t)}{\partial t}=\frac{1}{2 \hbar} \operatorname{Im}\left[\mathbf{P}_{v}(t) \cdot \mathbf{E}_{v}^{*}(t)\right] .
$$

Note that the self-energy matrix in (5c) does not contribute to the final result (see Sec. 16.2 of Ref. [19]). For a stationary pulse, Eq. (17) reduces further to the familiar expression [19]

$$
w_{\mathrm{exc}}=\frac{1}{2 \hbar}\left|\mathbf{E}_{\nu}\right|^{2} \operatorname{Im} \chi_{\mathrm{e}}(\omega)=\frac{I_{\nu} \kappa_{\mathrm{abs}}\left(\omega_{\nu}\right)}{\hbar \omega_{\nu}},
$$

with $I_{v} \equiv(c / 8 \pi)\left|\mathbf{E}_{v}\right|^{2}$ denoting the X-ray intensity.

The total energy of the material at time $t$, including its interaction with the $\mathrm{x}$-ray field, can be written as [16]

with

$$
\begin{aligned}
W_{v v \sigma} & \equiv \sum_{v^{\prime}=1}^{N_{\mathrm{MO}}} \sum_{\sigma^{\prime}=\alpha, \beta}\left(V_{v v v^{\prime} v^{\prime}}^{\sigma \sigma^{\prime}}-\delta_{\sigma \sigma^{\prime}} V_{v v^{\prime} v^{\prime} v}^{\sigma \sigma}\right) f_{v^{\prime} \sigma^{\prime}}(0)+\sum_{c=1}^{N_{\text {core }}} \sum_{\mu=1}^{N} \sum_{\sigma^{\prime}=\alpha, \beta} V_{v, v, c \mu, c \mu}^{\sigma \sigma^{\prime}} f_{c \mu \sigma^{\prime}}(0), \\
W_{c \mu, c \mu, \sigma} & \equiv \sum_{v=1}^{N_{\mathrm{MO}}} \sum_{\sigma^{\prime}=\alpha, \beta} V_{c \mu, c \mu, v, v}^{\sigma \sigma^{\prime}} f_{v \sigma^{\prime}}(0)+\sum_{c^{\prime}=1}^{N_{\text {core }}} \sum_{\mu^{\prime}=1}^{N} \sum_{\sigma^{\prime}=\alpha, \beta}\left(V_{c \mu, c \mu, c^{\prime} \mu^{\prime}, c^{\prime} \mu^{\prime}}^{\sigma \sigma^{\prime}}-\delta_{\sigma \sigma^{\prime}} \delta_{\mu \mu^{\prime}} V_{c \mu, c^{\prime} \mu, c^{\prime} \mu, c \mu}^{\sigma \sigma^{\prime}}\right) f_{c^{\prime} \mu^{\prime} \sigma^{\prime}}(0) .
\end{aligned}
$$

When we set $t=0$ in Eq. (19) and substitute the initial conditions (12), the resultant $\langle H(0)\rangle$ coincides with the UHF expression of the ground-state energy. To check the internal consistency of our formalism, let us calculate the instantaneous energy absorption rate per unit volume,

$$
w_{\text {ene }}(t) \equiv \frac{n_{\text {atom }}}{N} \frac{\partial\langle H(t)\rangle}{\partial t},
$$

by using Eqs. (5), (7), (11), and (19). If the contribution of Auger decay to $\langle H(t)\rangle$ is neglected, we arrive at an expression

$$
w_{\text {ene }}(t)=\hbar \omega_{\nu} w_{\text {exc }}(t)-\frac{1}{2} \operatorname{Im}\left[\frac{\partial \mathbf{E}_{v}^{*}(t)}{\partial t} \cdot \mathbf{P}_{\nu}(t)\right],
$$

where $w_{\text {exc }}(t)$ is given by Eq. (17). Clearly, the first term on the right-hand side of (22) can be interpreted as the photon energy $\hbar \omega_{v}$ absorbed per unit time; the second term, related to a variation of the pulse shape, has been confirmed to be negligibly small in the hard $\mathrm{x}$-ray regime treated in this work. Successful derivations of formulas (17) and (22) may justify our RDM equations (5a)-(5c).

\section{III. $K$-SHELL ABSORPTION OF COPPER}

On the basis of the formalisms described in Sec. II, we shall study the $K$-shell absorption spectra of copper and their dependence on laser intensities. The NDDO MO calculation is performed for a $\mathrm{Cu}_{19}$ cluster $(N=19)$ in face-centered-cubic configuration [12], which consists of 12 nearest-neighbor and 6 second-neighbor atoms surrounding a central atom. The nearest-neighbor distance is taken to be $R_{\mathrm{nn}}=2.55 \AA$, which corresponds to the bulk atomic density $n_{\text {atom }}=8.47 \times$ $10^{22} \mathrm{~cm}^{-3}$. The cluster contains $N_{\text {val }}^{\alpha}=105$ up-spin and $N_{\text {val }}^{\beta}=104$ down-spin valence electrons; the total number 


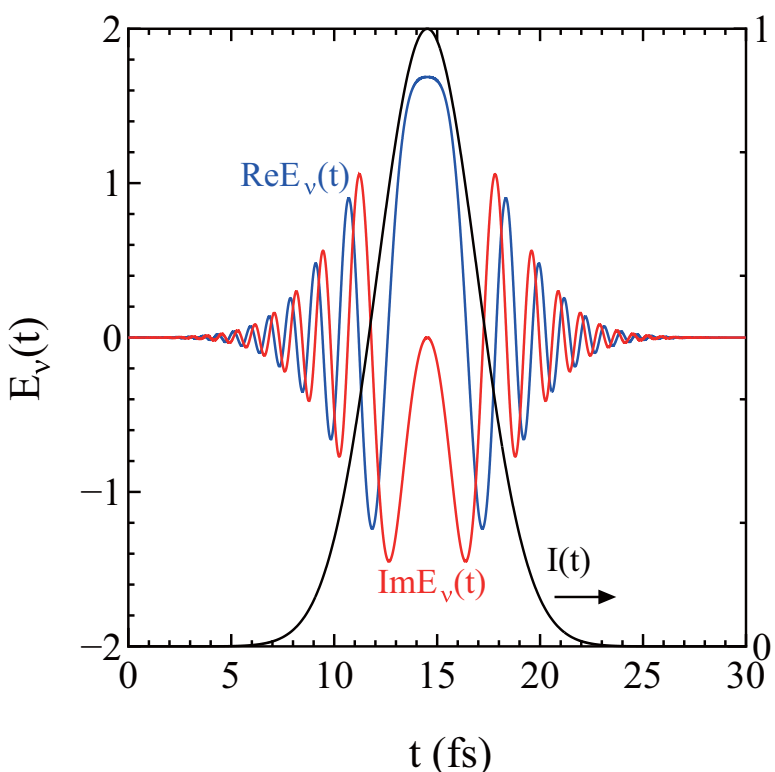

FIG. 1. Pulse shape $E_{v}(t)$ and intensity profile $I(t)$ of the x-ray electric field for $I_{v}=10^{17} \mathrm{~W} / \mathrm{cm}^{2}$.

of valence MO's is $N_{\mathrm{MO}}=399$. Computational details are given in Ref. [12]. The incident $\mathrm{x}$ ray is modelled by a linearly chirped Gaussian pulse [13] polarized along the [100] direction,

$$
E_{v}(t)=E_{0} \exp \left[-(1+i a)\left(\frac{t-t_{1}}{\tau_{\mathrm{G}}}\right)^{2}\right],
$$

with $a=9.95, \tau_{\mathrm{G}}=4.84 \mathrm{fs}$, and $t_{1}=3 \tau_{\mathrm{G}}$. The pulse duration, defined as the full width at half maximum (FWHM) of $\left|E_{v}(t)\right|^{2}$, is $\tau_{\mathrm{p}}=\sqrt{2 \ln 2} \tau_{\mathrm{G}}=5.7 \mathrm{fs}$. The FWHM $\Delta \omega_{\mathrm{p}}$ of the spectral profile $\left|\tilde{E}_{\nu}(\omega)\right|^{2}$ is $\Delta \omega_{\mathrm{p}}=\sqrt{8\left(1+a^{2}\right) \ln 2} / \tau_{\mathrm{G}}$, which amounts to $\hbar \Delta \omega_{\mathrm{p}}=3.2 \mathrm{eV}$. The parameter $a$ has been introduced to enlarge $\Delta \omega_{\mathrm{p}}$ so that the spectrum over a reasonably wide frequency range can be obtained for a single-shot pulse. The pulse shape $E_{v}(t)$ and the intensity profile $I(t) \equiv$ $(c / 8 \pi)\left|E_{v}(t)\right|^{2}$ are illustrated in Fig. 1 for the peak intensity $I_{v} \equiv(c / 8 \pi) E_{0}^{2}=10^{17} \mathrm{~W} / \mathrm{cm}^{2}$. The $\mathrm{x}$-ray carrier frequency is chosen as $\hbar \omega_{v}=8945 \mathrm{eV}$. It should be noted that the actual pulse shape of XFEL operating in the self-amplified spontaneous emission mode contains stochastic oscillations [20], which cannot be reproduced by a simple analytic formula such as Eq. (23). Simulation for such a rapidly fluctuating pulse is not pursued in this work, however, since it is beyond the applicability of the SVEA in Sec. II A.

We confine ourselves to $K$-shell excitation by setting $\mathbf{d}_{v, c \mu}^{\sigma} \equiv 0$ for $c \neq 1 s$. Since the Auger lifetime of a $K$-shell hole in a copper atom is dominated by the $K-L_{23} L_{23}$ process [17], we may set

$$
\begin{aligned}
\left.\frac{\partial f_{1 s \mu \sigma}(t)}{\partial t}\right]_{\text {Auger }} & =\frac{1}{\tau_{\text {Auger }}}\left[1-f_{1 s \mu \sigma}(t)\right] f_{2 p_{\gamma} \mu \sigma}(t), \\
\left.\frac{\partial f_{2 p_{\gamma} \mu \sigma}(t)}{\partial t}\right]_{\text {Auger }} & =-\frac{2}{3 \tau_{\text {Auger }}}\left[1-f_{1 s \mu \sigma}(t)\right] f_{2 p_{\gamma} \mu \sigma}(t) \\
\gamma & =x, y, z
\end{aligned}
$$

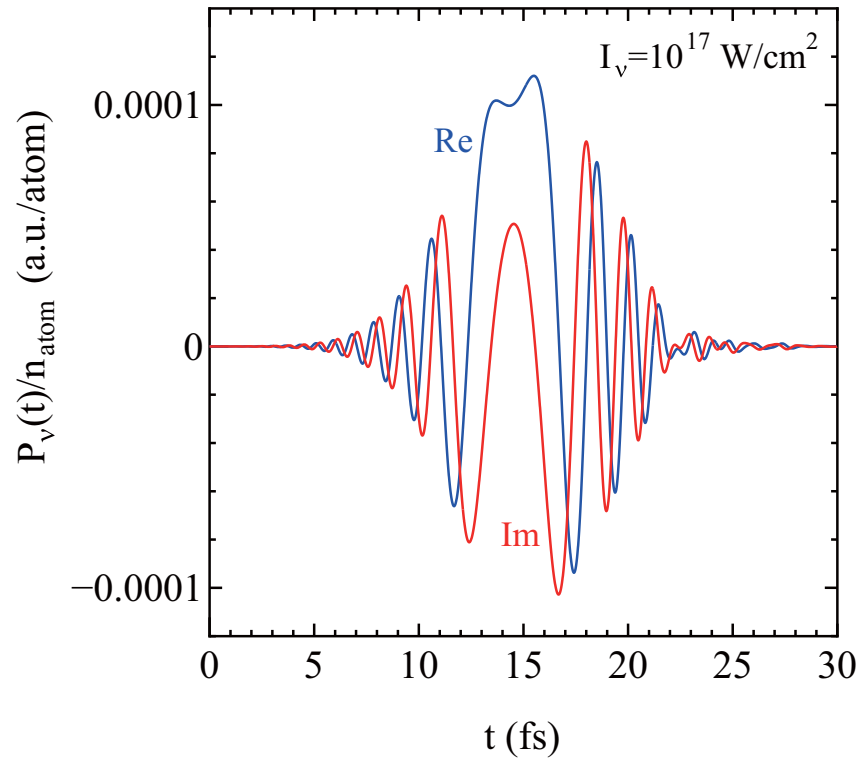

FIG. 2. Time evolutions of the electric polarization per atom for copper at $I_{v}=10^{17} \mathrm{~W} / \mathrm{cm}^{2}$. Real and imaginary parts are indicated by blue and red curves, respectively.

in Eq. (5b), where $f_{2 p_{x} \mu \sigma}(t)=f_{2 p_{y} \mu \sigma}(t)=f_{2 p_{z} \mu \sigma}(t)$ is assumed and the total $K-L L$ rate $1 / \tau_{\text {Auger }} \approx 2 \times 10^{-2}$ a.u. is adopted from the database [17]. Further cascade decay is neglected. Starting with the initial conditions (12), Eqs. (5a)(5c) have been integrated numerically using the midpoint method with $\hbar \Gamma=0.272 \mathrm{eV}$ and the time step $\Delta t=0.121$ as. The time evolutions of polarization (13) so computed are illustrated in Fig. 2 for $I_{v}=10^{17} \mathrm{~W} / \mathrm{cm}^{2}$. Figure 3 displays the absorption spectra near the $K$ edge computed on the basis of Eq. (16) for various values of the peak intensity. The spectrum at low intensities $\left(I_{v}<10^{15} \mathrm{~W} / \mathrm{cm}^{2}\right)$ agrees with the linear-response result studied earlier [12]. We find that the

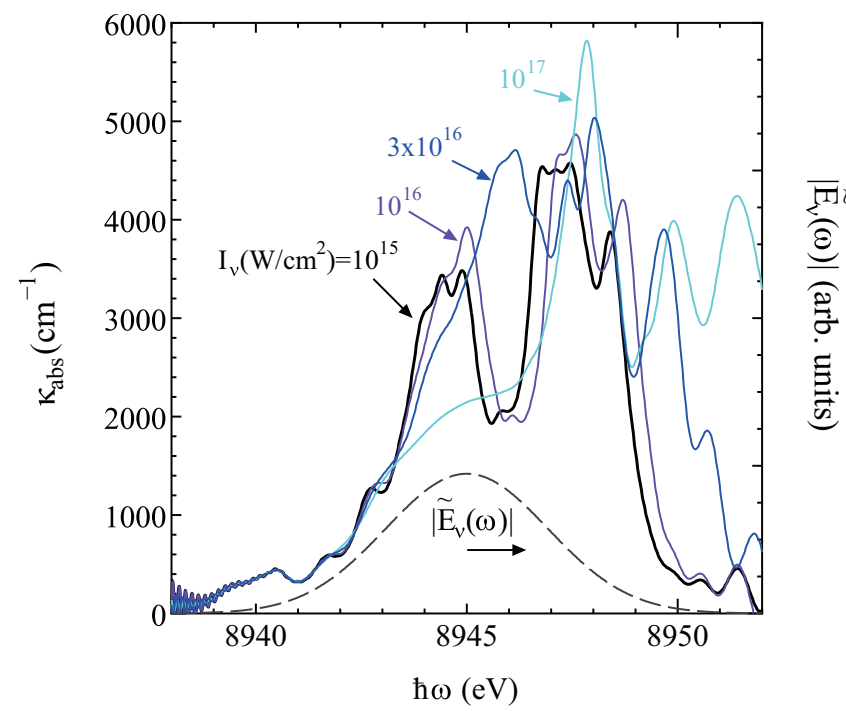

FIG. 3. $K$-shell absorption spectra of copper for various pulse peak intensities. The spectrum of the electric-field envelope (14) is superimposed by the dashed curve. 


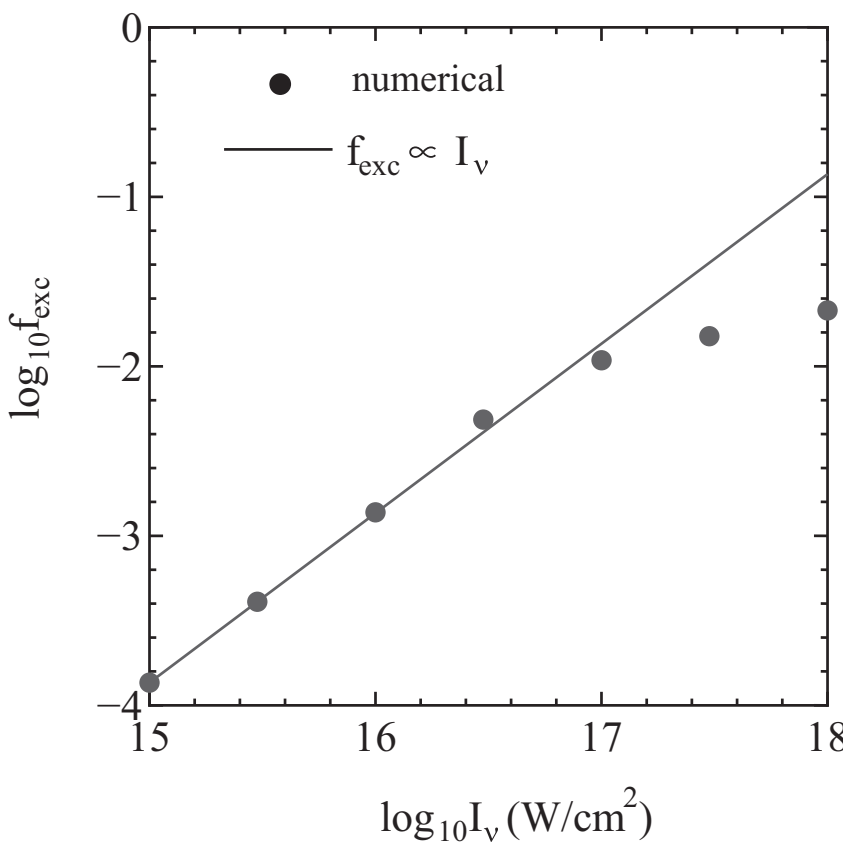

FIG. 4. The fraction of excited electrons in copper as a function of pulse peak intensity. Filled circles represent numerical results. Solid line is a linear fit $f_{\text {exc }} \propto I_{v}$ valid in the low- $I_{v}$ limit.

spectrum above the $K$-edge (about $8943 \mathrm{eV}$ ) shifts to higher energies as the intensity increases. At the highest intensity $\left(I_{v}=10^{17} \mathrm{~W} / \mathrm{cm}^{2}\right)$, the originally sharp $K$ edge becomes broadened.

As mentioned in [12], the present cluster model is not accurate enough to reproduce the known absorption spectra of bulk copper [7]: The peaks and dips in the theoretical spectra are absent in experimental data [7] and considered as artifacts due to an incompleteness of the basis set and the pseudospectral treatment of the continuum [12]. The theoretical $K$-edge energy is also slightly underestimated compared to the experimental value, $8.98 \mathrm{keV}$ [7]. Nevertheless, the intensity dependence of the spectrum in Fig. 3 is clear and meaningful. Recent advances in theoretical methods to compute core-valence excitation spectra of atoms and molecules are summarized in [21] and references therein; none of them have reproduced intensity dependence of the spectra observed experimentally [7], however.

In Fig. 4, we plot the excitation degree $f_{\text {exc }}$ defined as the fraction of excited electrons, $f_{\text {exc }} \equiv$ $\sum_{v=1}^{N_{\mathrm{MO}}} \sum_{\sigma=\alpha, \beta}\left[f_{v \sigma}(\infty)-f_{v \sigma}(0)\right]$. In the region $I_{v}<$ $10^{17} \mathrm{~W} / \mathrm{cm}^{2}$, the linear relationship $f_{\text {exc }} \propto I_{\nu}\left(\propto E_{0}^{2}\right)$ can be observed, which means that the population change is second order in the electric field; the resultant change in the absorption spectrum is a third-order effect. A departure from the linear relationship can be found at $I_{v}>10^{17} \mathrm{~W} / \mathrm{cm}^{2}$, indicating a saturation of absorption. We may thus regard the region $I_{v}<10^{17} \mathrm{~W} / \mathrm{cm}^{2}$ as the third-order nonlinear regime.

Figure 5 illustrates time evolutions of $1 s$ and $2 p$ levels of a central atom in the cluster for $I_{v}=10^{17} \mathrm{~W} / \mathrm{cm}^{2}$. The population of the $1 \mathrm{~s}$ level initially decreases due to X-ray excitation but is soon refilled through the Auger decay. Since only the $K-L_{23} L_{23}$ process is taken into account, the core

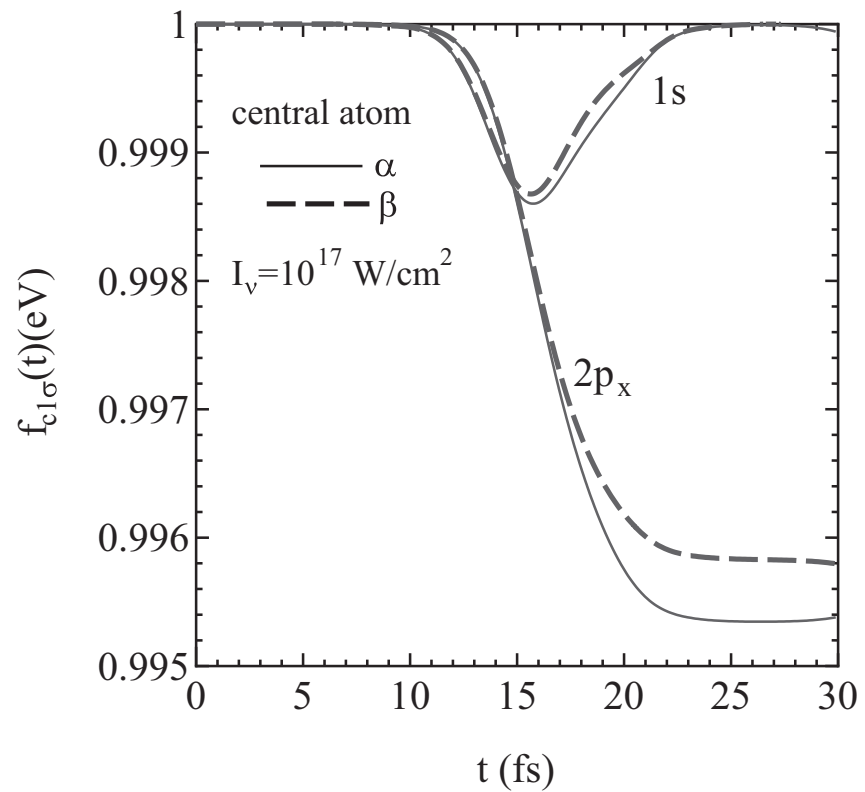

FIG. 5. Time evolutions of $1 s$ - and $2 p_{x}$-level populations of a central copper atom for $I_{v}=10^{17} \mathrm{~W} / \mathrm{cm}^{2}$.

hole eventually resides in $2 p$ orbitals for $t \rightarrow \infty$. We remark that our analysis of the competition between excitation and decay indicated in Fig. 5 is based on rate equations (24); a full wave-function-based analysis of related phenomena for molecules can be found in [22].

We find through comparison between Figs. 3-5 that an excitation fraction of only $1 \%$ is sufficient to induce a blueshift of the absorption spectrum by about a few eV. The origin of the spectral shift can be traced to the negative renormalization factor $\left(\tilde{\mathcal{E}}_{1 s \mu, 1 s \mu}^{\sigma}(t)\right)_{0}<0$ in Eq. (5a), which acts to increase the threshold energy of $K$-shell excitation. To see this, we show in Fig. 6 the renormalized $1 s$ energy level [14],

$$
\tilde{\mathcal{E}}_{1 s, \mu, \sigma}(t) \equiv \varepsilon_{1 s, \mu, \sigma}+\left(\tilde{\mathcal{E}}_{1 s \mu, 1 s \mu}^{\sigma}(t)\right)_{0},
$$

at $I_{v}=10^{17} \mathrm{~W} / \mathrm{cm}^{2}$ for the central atom $(\mu=1)$ in the cluster. It can be seen that $\tilde{\varepsilon}_{1 s, 1, \sigma}(t)$ is lowered by nearly $4 \mathrm{eV}$ as the $\mathrm{x}$-ray pulse passes through the cluster. In Fig. 7, we depict the asymptotic values of (25) for all atoms in the cluster. As the intensity increases, the energy levels $\tilde{\varepsilon}_{1 s, \mu, \sigma}(t \rightarrow \infty)$ are lowered and spread into a band; these features account for the blueshift of the spectra and broadening of the $K$ edge, respectively, in Fig. 3. The energy spread reflects the fact that $\left(\tilde{\mathcal{E}}_{1 s \mu, 1 s \mu}^{\sigma}(t)\right)_{0}$ depends on both $\mu$ and $\sigma$. We observe in Fig. 3 that the shift and broadening of the $K$ edge result in net reduction of absorption, which is thought to be the reason for the saturation of absorption above $10^{17} \mathrm{~W} / \mathrm{cm}^{2}$ in Fig. 4. The renormalized energy levels of low-lying unoccupied valence MO's $\tilde{\varepsilon}_{v \sigma}(t) \equiv \varepsilon_{v \sigma}+\left(\tilde{\mathcal{E}}_{v v}^{\sigma}(t)\right)_{0}$ for $t \rightarrow \infty$ are likewise exhibited in Fig. 8. Comparison between Figs. 7 and 8 reveals that the magnitude of valence-level lowering $\left(\tilde{\mathcal{E}}_{v v}^{\sigma}(t)\right)_{0}$ is significantly smaller than that of core-level lowering $\left(\tilde{\mathcal{E}}_{c \mu, c \mu}^{\sigma}(t)\right)_{0}$.

Tamasaku et al. [7] measured $K$-edge absorption spectra of copper by performing foil transmission experiments using SACLA XFEL with $\hbar \omega_{v}=8.99 \mathrm{keV}$ and $\tau_{\mathrm{p}} \approx 8 \mathrm{fs}$. They demonstrated that, as the X-ray intensity increases up to about 


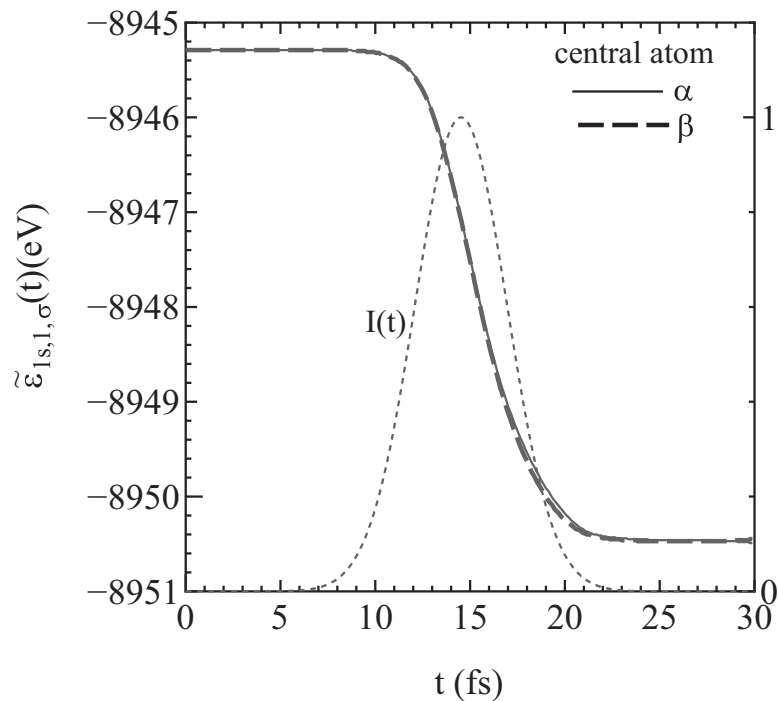

FIG. 6. Time evolutions of the renormalized $1 s$ energy levels $\tilde{\varepsilon}_{1 s, 1, \sigma}(t)$ based on Eq. (25) for a central copper atom at $I_{v}=$ $10^{17} \mathrm{~W} / \mathrm{cm}^{2}$. Solid and dashed curves correspond to up-spin $(\sigma=$ $\alpha)$ and down-spin $(\sigma=\beta)$ states, respectively. The dotted curve represents the intensity profile.

$10^{17} \mathrm{~W} / \mathrm{cm}^{2}$, the spectrum above the $K$ edge deviates toward the high-energy side, while the pre-edge region remains virtually unaltered (see Fig. 4 of Ref. [7]). It is remarkable that these features are qualitatively consistent with our prediction in Fig. 3.

\section{DISCUSSION}

In this section, physical mechanisms of the third-order nonlinearity found for copper in Sec. III are examined from a more general viewpoint. There are two contributions to

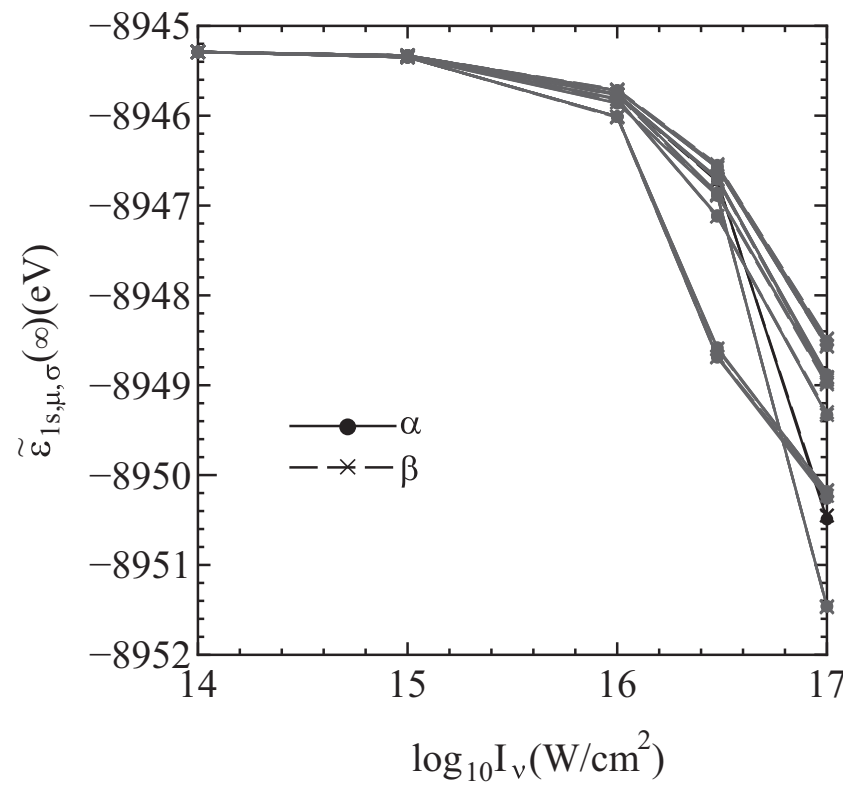

FIG. 7. Renormalized $1 s$ orbital energies (25) for copper at $t \rightarrow$ $\infty$ as functions of the pulse peak intensity.

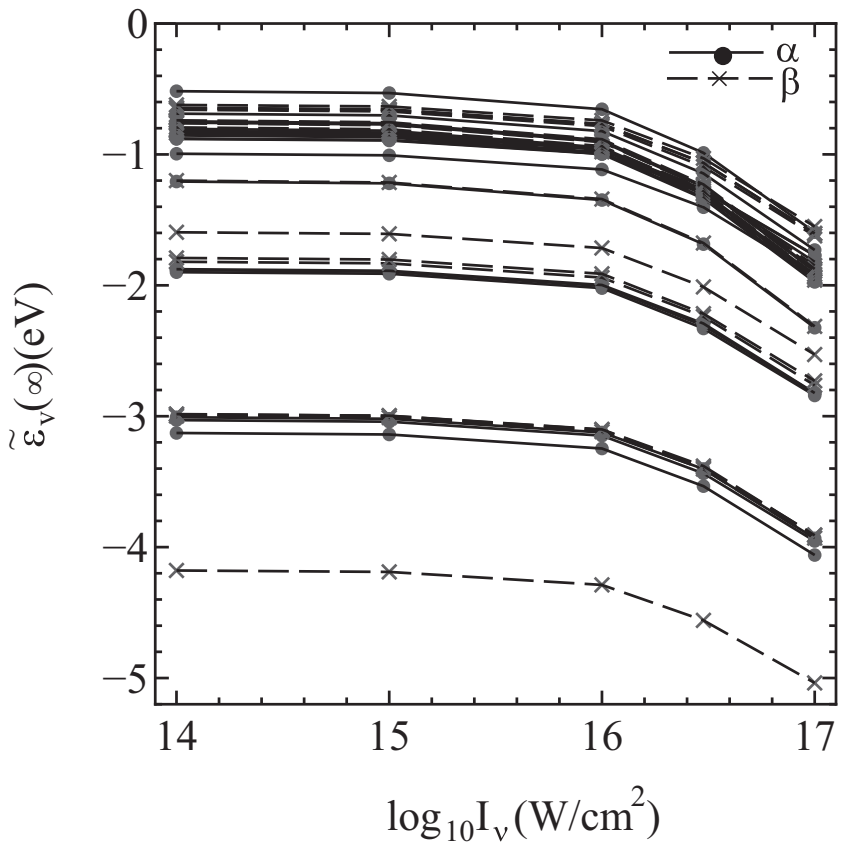

FIG. 8. Renormalized orbital energies of low-lying unoccupied valence states for copper at $t \rightarrow \infty$ as functions of the pulse peak intensity.

the third-order nonlinearity in Eq. (5a): the energy renormalization factor $\left(\tilde{\mathcal{E}}_{v v}^{\sigma}(t)\right)_{0}-\left(\tilde{\mathcal{E}}_{c \mu, c \mu}^{\sigma}(t)\right)_{0}$ and the population inversion factor $f_{c \mu \sigma}(t)-f_{v \sigma}(t)$. The latter quantity, coupled directly with the optical field, is the usual source of saturable absorption in nonlinear optics [1]. In the third-order nonlinear regime treated in Sec. III, however, it turns out that the difference between $f_{c \mu \sigma}(t)-f_{v \sigma}(t)$ and $f_{c \mu \sigma}(0)-f_{v \sigma}(0)$ is less than 0.01 so that the contribution from the population inversion factor is negligible. Bulk of the nonlinearity thus arises from $\left(\tilde{\mathcal{E}}_{c \mu, c \mu}^{\sigma}(t)\right)_{0}$. In the summation on the right-hand side of Eq. (11a), the quantity of particular relevance is the on-site Coulomb repulsion $U_{c c^{\prime}} \equiv V_{c \mu, c \mu, c^{\prime} \mu, c^{\prime} \mu}^{\sigma \sigma^{\prime}}$ between two electrons in core orbitals $c$ and $c^{\prime}$ on the same atom, whose magnitude can be considerably large [23]; we estimate that $U_{1 \mathrm{~s} 1 \mathrm{~s}}=483.5 \mathrm{eV}$ and $U_{1 \mathrm{~s} 2 \mathrm{p}}=163.8 \mathrm{eV}$ for $1 s$ and $2 p$ orbitals in copper. When a down-spin electron in a $1 s$ orbital is excited, the net repulsive force exerted on the up-spin electron in the same orbital is reduced, resulting in a negative energy renormalization $\left(\tilde{\mathcal{E}}_{1 s \mu, 1 s \mu}^{\alpha}(t)\right)_{0} \approx U_{1 s 1 s}\left[f_{1 s \mu \beta}(t)-f_{1 s \mu \beta}(0)\right]<$ 0 . The $K$-shell holes are transferred rapidly to the $L$ shell through the $K-L L$ Auger process, which again yields a negative shift of the $1 s$ level, $\left(\tilde{\mathcal{E}}_{1 s \mu, 1 s \mu}^{\sigma}(t)\right)_{0} \approx$ $U_{1 s 2 p} \sum_{\gamma=x, y, z} \sum_{\sigma^{\prime}=\alpha, \beta}\left[f_{2 p_{\gamma} \mu \sigma^{\prime}}(t)-f_{2 p_{\gamma} \mu \sigma^{\prime}}(0)\right]<0$; its magnitude can easily exceed $1 \mathrm{eV}$ even if the average number of core holes per atom is as small as 0.01 . Thus, the spectral shift on the order of a few eV can naturally be accounted for by the core-level renormalization effect.

The intraatomic energy renormalization effect mentioned above is irrespective of the atomic density and hence may be observable in small molecules and gaseous targets as well. To confirm this point numerically, we have repeated analogous calculations for an isolated $\mathrm{Cu}_{2}$ dimer $(N=2)$ by adopting the experimental bond length $R_{\mathrm{nn}}=2.22 \AA$ [24] and the 


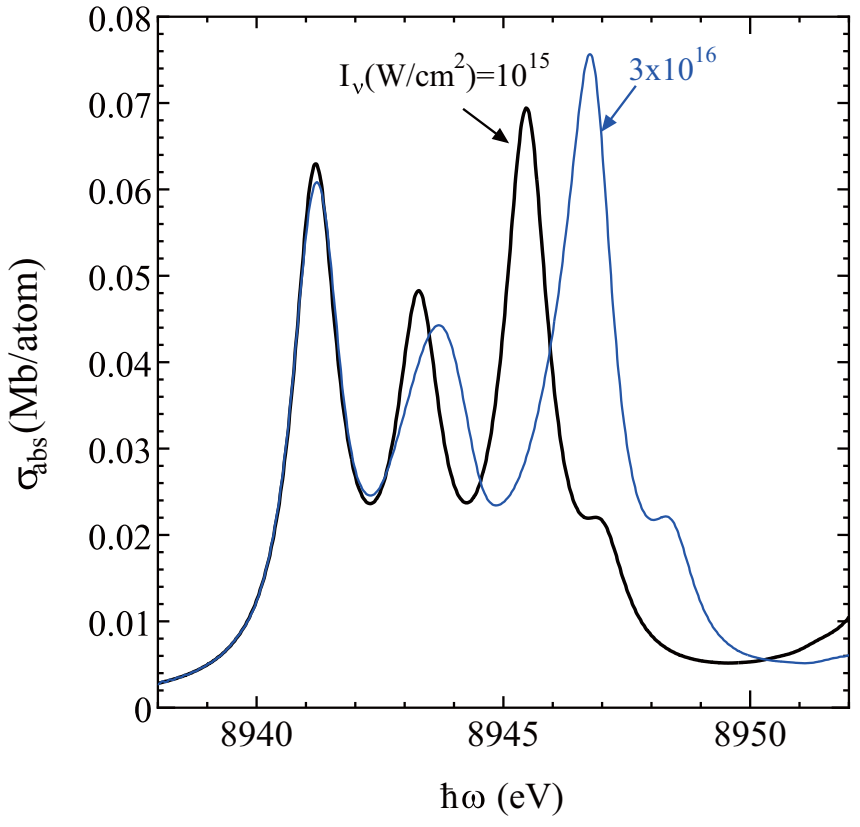

FIG. 9. $K$-shell absorption cross sections of a $\mathrm{Cu}_{2}$ dimer at $I_{v}=$ $10^{15}$ and $3 \times 10^{16} \mathrm{~W} / \mathrm{cm}^{2}$.

damping parameter $\hbar \Gamma=0.544 \mathrm{eV}$; the direction of $\mathbf{E}_{v}(t)$ is perpendicular to the molecular axis. The resultant absorption spectra are shown in Fig. 9. A blueshift of the spectrum similar to Fig. 3 is indeed predicted above $8943 \mathrm{eV}$ as the $\mathrm{x}$-ray intensity increases. In condensed phases, the contributions from neighboring core holes $\left(\mu^{\prime} \neq \mu\right)$ and valence electrons in Eq. (11a) also come into play. It should be noted on the right-hand side of Eq. (5a) that the renormalization factor $\left(\tilde{\mathcal{E}}_{v v}^{\sigma}(t)\right)_{0}-\left(\tilde{\mathcal{E}}_{c \mu, c \mu}^{\sigma}(t)\right)_{0}$ depends on time and acts to modulate the bare excitation frequency $\left(\varepsilon_{v \sigma}-\varepsilon_{c \mu \sigma}\right) / \hbar$ dynamically; it does not bring about a simple constant shift of the absorption spectrum when Fourier-transformed into the frequency space.

A salient feature shown in the high-intensity region of Fig. 7 is the spread of core orbital energies, which causes a broadening of the absorption edge, leading to saturation of absorption. Physical origin of the energy-level spread is not trivial but can be traced to the $\mu$ and $\sigma$ dependence of $\left(\tilde{\mathcal{E}}_{c \mu, c \mu}^{\sigma}(t)\right)_{0}$ in $(25)$. The $\mu$ dependence of $\left(\tilde{\mathcal{E}}_{c \mu, c \mu}^{\sigma}(t)\right)_{0}$ implies that the excitation proceeds locally at a different rate depending on the atomic position. Such an inhomogeneous excitation may generally occur when a core hole created locally in the crystal breaks the translational symmetry. The $\sigma$ dependence of $\left(\tilde{\mathcal{E}}_{c \mu, c \mu}^{\sigma}(t)\right)_{0}$ indicates an imbalance between the excitation dynamics of the two electrons within the same core orbital. This is the case in usual atomic ionization from a doubly occupied orbital, where one of the electrons is ionized favorably over the other [25]; spin-unrestricted formalism is essential for treating such a selective excitation. The possibility of a spontaneous emergence of such a "symmetry breaking" in correlated electron-hole dynamics remains to be investigated further in future works.

Tamasaku et al. [7] argued that collisional relaxation of hot electrons created by XFEL might be responsible for the observed spectral shift, while theoretical spectral shift in Sec. III has been predicted from core-level renormalization alone. Generally, hot electron relaxation in a metal accompanies low-energy excitation of conduction electrons across the Fermi level [26], so the absorption may be enhanced below the edge and reduced above the edge [5]; such a feature has been observed for pulse duration longer than ps in the case of visible light [5]. For a femtosecond x-ray pulse, hot-electron effects may be observable at sufficiently high x-ray intensities where $f_{\text {exc }}$ becomes appreciably large. In the RDM formalism, dynamics of hot electrons in the valence band can be taken into account by adding a collision term on the right-hand side of Eq. (5c) [26].

The blueshift of the spectrum predicted in this work may be understood as an effective enhancement of the core-valence band gap [19]. This is a feature opposite to the case of valence electron excitation in semiconductors, where negative exchange energy of the electron-hole plasma yields band-gap reduction (see, e.g., Chap. 15 of Ref. [19]). Both phenomena are the consequences of Coulombic renormalization effects and accounted for by the self-energy matrices in RDM equations, with the only difference lying in the spatial extension of the electronic orbitals [23].

The single-particle mean-field approach as adopted in this work describes core-hole formation and decay in a probabilistic way by fractional occupation numbers. Validity of such a mean-field description is not trivial and should be checked for a low-density atomic gas, for instance, by diagonalizing the atomic Hamiltonian exactly and representing the density matrix in terms of the exact atomic eigenstates. In such a representation, a quantum-mechanical expression for the third-order susceptibility is formally available $[1,2]$, but it contains cumbersome summations over virtual transitions to intermediate states whose treatment requires extra care; simplified models such as two- or three-level atoms are frequently used [1]. We speculate that, if density-matrix equations analogous to (5) can be formulated for multilevel "atoms" containing core holes and ionization continuum, they might give a clue to the origin of third-order x-ray nonlinearity from the viewpoint beyond the mean-field approximation. The resultant equations will not contain Coulomb matrix elements (9) since the electron-electron interactions in each atom are all incorporated into the eigenenergies and eigenfunctions of the atom itself. Elucidation of mutual correspondence between the single-particle and multilevel-atom formalisms should await for further studies in the future. It also remains to be seen whether the conventional anharmonic oscillator models in nonlinear optics [4] can be extended to X-ray regime so as to put physical interpretations on the nonlinear susceptibilities obtained numerically in this work.

\section{CONCLUDING REMARKS}

In conclusion, we have presented RDM equations for resonant core-valence electron excitation by an intense femtosecond x-ray pulse. Through numerical solutions to the RDM equations in the cluster model, intensity dependence of $K$ shell absorption spectra for metallic copper has been studied. We have thereby shown that third-order nonlinearity stems from a negative renormalization of the core orbital energy due to strong attractive electron-hole interaction in a localized core orbital. Such a Coulomb-induced nonlinearity surpasses 
the hitherto considered mechanisms based on the population inversion factors. As the intensity increases, the renormalized core orbital energies are lowered and spread into a band, leading to a blueshift and edge broadening of the absorption spectra. These features agree qualitatively with experimental results for copper obtained by SACLA XFEL. Broadening of the absorption edge in turn causes saturation of absorption, which can be expected even if the degree of electron excitation is only a few percent. In order for extension of Eqs. (5) beyond the third-order nonlinear regime, we would have to consider additional effects such as hot-electron relaxation, orbital deformation, and interference effects due to core-hole decay [27].

\section{ACKNOWLEDGMENTS}

The author is grateful to Dr. Y. Inubushi, Dr. K. Tamasaku, and Dr. H. Yoneda for valuable discussions. This work was supported in part through JSPS KAKENHI Grants No. 16H02118 and No. 18H03474.
[1] R. W. Boyd, Nonlinear Optics (Academic, San Diego, CA, 1992), Chap. 4.

[2] M. Sheik-Bahae and M. P. Hasselbeck, in Handbook of Optics, edited by M. Bass, J. M. Enoch, E. W. Van Stryland, and W. L. Wolfe (McGraw-Hill, New York, 2001), Vol. IV, Chap. 17.

[3] F. Bassani and S. Scandolo, Phys. Rev. B 44, 8446 (1991).

[4] M. S. Kim and C. S. Yoon, Phys. Rev. Lett. 93, 203903 (2004).

[5] R. W. Boyd, Z. Shi, and I. De Leon, Opt. Commun. 326, 74 (2014).

[6] M. Conforti and G. Della Valle, Phys. Rev. B 85, 245423 (2012).

[7] K. Tamasaku, E. Shigemasa, Y. Inubushi, I. Inoue, T. Osaka, T. Katayama, M. Yabashi, A. Koide, T. Yokoyama, and T. Ishikawa, Phys. Rev. Lett. 121, 083901 (2018).

[8] H. Yoneda, Y. Inubushi, M. Yabashi, T. Katayama, T. Ishikawa, H. Ohashi, H. Yumoto, K. Yamauchi, H. Mimura, and H. Kitamura, Nat. Commun. 5, 5080 (2014).

[9] D. S. Rackstraw, O. Ciricosta, S. M. Vinko, B. Barbel, T. Burian, J. Chalupský, B. I. Cho, H.-K. Chung, G. L. Dakovski, K. Engelhorn, V. Hájková, P. Heimann, M. Holmes, L. Juha, J. Krzywinski, R. W. Lee, S. Toleikis, J. J. Turner, U. Zastrau, and J. S. Wark, Phys. Rev. Lett. 114, 015003 (2015).

[10] F. de Groot and A. Kotani, Core Level Spectroscopy of Solids (CRC, Boca Raton, FL, 2008), Chap. 3.

[11] Electronic Properties of Solids Using Cluster Methods, edited by T. A. Kaplan and S. D. Mahanti (Springer, New York, 1995).
[12] H. Kitamura, J. Phys. B: At. Mol. Opt. Phys. 53, 095602 (2020).

[13] J.-C. Diels and W. Rudolph, Ultrashort Laser Pulse Phenomena, 2nd ed. (Academic, Burlington, 2006), Chap. 1.

[14] H. Kitamura, Int. J. Quantum Chem. 117, 25442 (2017).

[15] V. L. Jacobs, Phys. Rev. A 90, 033841 (2014).

[16] H. Kitamura, Int. J. Quantum Chem. 114, 1518 (2014).

[17] D. L. Walters and C. P. Bhalla, At. Data 3, 301 (1971).

[18] E. H. S. Burhop and W. N. Asaad, Adv. At. Mol. Phys. 8, 163 (1972).

[19] H. Haug and S. W. Koch, Quantum Theory of the Optical and Electronic Properties of Semiconductors (World Scientific, Singapore, 2003).

[20] T. Pfeifer, Y. Jiang, S. Dusterer, R. Moshammer, and J. Ullrich, Opt. Lett. 35, 3441 (2010).

[21] P. Norman and A. Dreuw, Chem. Rev. 118, 7208 (2018).

[22] E. Pahl, H.-D. Meyer, and L. S. Cederbaum, Z. Phys. D 38, 215 (1996).

[23] H. Kitamura, Europhys. Lett. 94, 27005 (2011).

[24] M. D. Morse, Chem. Rev. 86, 1049 (1986).

[25] K. Burnett, J. B. Watson, A. Sanpera, and P. L. Knight, Philos. Trans. R. Soc. London, Ser. A 356, 317 (1998).

[26] H. Kitamura, J. Electron. Spectrosc. Relat. Phenom. 232, 45 (2019).

[27] P. V. Demekhin and L. S. Cederbaum, Phys. Rev. A 83, 023422 (2011). 